\title{
TB-HIV : Therapeutic challenge from two deadly comrades
}

\section{Rawshan Arra Khanam}

TB is the ninth leading cause of death worldwide and the leading cause from a single infectious agent, ranking above HIV/AIDS. In 2016, there were an estimated 1.3 million TB deaths among HIV-negative people (down from 1.7 million in 2000) and an additional 374000 deaths among HIV-positive people. An estimated 10.4 million people fell ill with TB in 2016: $90 \%$ were adults, $65 \%$ were male, $10 \%$ were people living with $\mathrm{HIV}$ ( $74 \%$ in Africa) and $56 \%$ were in five countries: India, Indonesia, China, the Philippines and Pakistan. Multidrug-resistant TB (MDR-TB) remains a public health crisis and a health security threat. WHO estimates that there were 600000 new cases with resistance to rifampicin the most effective first-line drug, of which 490000 had MDR-TB ${ }^{1}$.

High clinical suspicion plays a vital role in diagnosing early TB disease especially in with advanced HIV due to lack of classical symptoms. Line probe assays (LPA), endorsed by the WHO in 2008, are highly sensitive ( $\geq 97 \%$ ) and specific ( $\geq 99 \%$ ) for the detection of rifampicin resistance, alone or in combination with isoniazid (sensitivity $\geq 90 \%$; specificity $\geq$ $99 \%$ ), but it is used in smear-positive sputum specimens only $^{2}$. Xpert-MTB rif (GeneXpert) though detect rifampicin resistance only, can be used in smear negative patients also ${ }^{3}$. Xpert-Ultra is an improved version that has been equated to a liquid culture, able to detect mycobacterium tuberculosis as well as rifampicin resistance even in smear negative HIV patients where the conventional Xpert-MTB rif has a lesser yield ${ }^{4}$.

As the risk of TB developing in HIV infected individuals is $5-10 \%$ every year, current WHO guidelines recommend screening of all HIV-infected individuals for TB (intensified case finding), and if found to be uninfected, receive isoniazid preventive therapy (IPT) for a 6 months, irrespective of Tuberculin Skin Test(TST) status ${ }^{5,6}$.

HIV infection favours mycobacteremia and tissue invasion resulting in abundance of intracellular and intermittently dividing bacilli, making rifampicin indispensable in HIV associated $\mathrm{TB}^{7}$.

Management of pulmonary TB in HIV may be complicated by emergence of drug resistance, Immune reconstitution inflammatory syndrome (IRIS), drug-drug interaction etc. demands meticulous monitoring ${ }^{8}$. TB treatment duration is not influenced or confounded by HIV infection currently being 6 months for Pulmonary and extended in severe forms of extra pulmonary TB like bone and neurological TB. Centre for Disease Control, Atlanta recommends extension of anti-tubercular therapy (ATT) beyond 6 months in HIV-coinfected pulmonary TB patients in specific instances like delayed sputum conversion or poor clinical improvement with/without evidence of dissemination, low CD4 count at nadir and presence of cavitation ${ }^{9}$.

The greater percentage of persistors and bacillary mutants in HIV coinfection facilitates and favours emergence of drug resistance to ATT notably rifampicin. Acquired rifamycin resistance (ARR) is the emergence of resistance (defined as MIC > $128 \mu \mathrm{g} / \mathrm{ml}$ ) to rifamycin among patients whose pretreatment isolates were sensitive. ARR is a rarity in HIV seronegative individuals with pulmonary TB (PTB). The proportion of recurrences due to reinfection is more frequent in HIV positive individuals especially in countries with a higher TB burden than HIV-seronegative individuals with TB who have a true relapse ${ }^{10}$.

Causes of true failures in HIV associated TB include emergence of ATT drug resistance, virological failure to ART, immunological discordance (lower CD4 with undetectable viral load) and malabsorption of drugs leading to cryptic non-adherence ${ }^{11}$. Treatment for drug resistant-TB consists of at least 4-5 effective drugs. The treatment success rate for HIV-associated TB (2015 cohort) was 78\% and for extensively drug-resistant TB (XDRTB) (2014 cohort) it was $30 \%$. At least 35 countries have introduced shorter regimens for treatment of MDR/RR-TB. As part of efforts to improve outcomes for MDR/XDR-TB, 89 countries and territories had started using bedaquiline and 54 had used delamanid by June 2017.

Recently a 9 to 12-month regimen (known as the 'Bangladesh regimen') proved to be effective in treating MDR-TB cases. It included an initial phase of 4 to 6 months of kanamycin, moxifloxacin, prothionamide, clofazimine, pyrazinamide, high-dose isoniazid, and ethambutol, followed by 5 months of moxifloxacin, clofazimine, pyrazinamide, and ethambutol ${ }^{12}$. Evidence for this regimen originated from the Bangladesh observational cohort study by Van Deun among MDR-TB patients which showed a relapse free cure rate of $84.5 \%$ among 515 patients, but in a virtually HIV-free population ${ }^{13}$. The same regimen, tested in the fran-cophone African countries, among 408 patients (that included HIV positive $22 \%$ ) showed a relapse free survival rate of $82.1 \%$. Although treatment success rates did not differ by HIV status among those who survived, the death rate was higher among HIV co-infected $18 \%$ died, compared to $5 \%$ in HIV-seronegative patients ${ }^{14}$. 
Our aim should be to combine the ideal anti-tuberculosis treatment (ATT) with mutually compatible highly active antiretroviral therapy (HAART) combinations to save millions of lives and also offer a better quality of life to patients suffering from this coinfection.

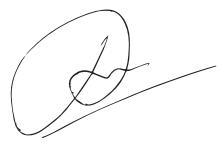

Dr. Rawshan Arra Khanam MBBS, MD (Chest Diseases)

Jr. Consultant, Dept. of Respiratory Medicine

United Hospital Ltd.

Gulshan 2, Dhaka

Contact : +88 01843894436

Email : rawshan.dr@gmail.com

\section{References:}

1. World Health Organization. Global tuberculosis report 2017. WHO /HTM/ TB/ 2017. 23 GENEVA: WHO;2017

2. Molecular line probe assays for rapid screening of patients at risk of multidrug-resistant tuberculosis. policy statement. Geneva: WHO; 2008.

3. World Health Organization. Automated real-time nucleic acid amplifica-tion technology for rapid and simultaneous detection of tuberculosis and rifampicin resistance: Xpert MTB/RIF system: policy statement. Geneva: World Health Organization; 2011.

4. Alland D, Rowneki M, Smith L. Xpert MTB/RIF Ultra: a new near-patient TB test with sensitivity equal to culture abstract 91 CROI 2015, Seattle.
5. Kaplan JE, Benson C, Holmes KH. Guidelines for prevention and treatment of opportunistic infections in HIV-infected adults and ado-lescents: recommendations from $\mathrm{CDC}$, the National Institutes of Health, and the HIV Medicine Association of the Infectious Diseases Society of America. MMWR Recomm Rep. 2009;58(RR-4):1-207.

6. World Health Organization. Guidelines for intensified tuberculosis case-finding and isoniazid preventive therapy for people living with HIV in resource-constrained settings. Geneva: World Health Organization. 2011.

7. Mitchinson DA. Role of individual drugs in the chemotherapy of tuber-culosis. Int J Tuberc Lung Dis. 2000;4(9):796-806.

8. Burman WJ, Jones B. Treatment of HIV-related tuberculosis in the era of effective antiretroviral therapy. AJRCC. 2001;164(1):7-12.

9. Centre for Disease Control. Guidelines for prevention and treatment of opportunistic infections in HIV-infected adults and adolescents. Am Thorac Soc MMWR. 2009;58:1-198

10. Narayanan S, Swaminathan S, Supply P. Impact of HIV infection on the recurrence of tuberculosis in South India. J Infect Dis. 2010;201(5):691-703

11. Nahid P, Gonzalez LC, Rudoy I. Treatment outcomes of patients with HIV and tuberculosis. Am J RespirCrit Care Med. 2007;175(11):1199-206

12. Giovanni S., Simon T., Rosella C., Lia D'A, Zhenia F., Alimuddin Z. et al. Applicability of the shorter 'Bangladesh regimen' in high multidrug-resistant tuberculosis settings.International Journal of Infectious Diseases 56 (2017) 190-193

13. Aung KJM, Van Deun A, Declercq E. Successful '9-month Bang-ladesh regimen' for multidrugresistant tuberculosis among over 500 consecutive patients. Int $\mathrm{J}$ Tuberc Lung Dis. 2014;18(10):1180-7.

14. Kuaban, et al. West African Cohort study. Late breaker session. 46th Union World Conference on Lung Health, Cape Town.2-5 Dec 2015 